\title{
Simulation of non-dilute fibre suspensions using RBF-based macro-micro multiscale method
}

\author{
Hung Quoc Nguyen ${ }^{1} \cdot$ Canh-Dung Tran ${ }^{1}$
}

Received: 24 September 2021 / Revised: 20 January 2022 / Accepted: 24 January 2022 / Published online: 25 February 2022

(c) The Author(s) 2022

\begin{abstract}
The multiscale stochastic simulation method based on the marriage of the Brownian Configuration Field (BCF) and the Radial Basis Function mesh-free approximation for dilute fibre suspensions by our group, is further developed to simulate non-dilute fibre suspensions. For the present approach, the macro and micro processes proceeded at each time step are linked to each other by a fibre contributed stress formula associated with the used kinetic model. Due to the feature of non-dilute fibre suspensions, the interaction between fibres is introduced into the evolution equation to determine fibre configurations using the BCF method. The fibre stresses are then determined based on the fibre configuration fields using the Phan-ThienGraham model. The efficiency of the simulation method is demonstrated by the analysis of the two challenging problems, the axisymmetric contraction and expansion flows, for a range of the fibre concentration from semi-dilute to concentrated regimes. Results evidenced by numerical experiments show that the present method would be potential in analysing and simulating various suspensions in food and medical industries.
\end{abstract}

Keywords Brownian configuration field · Radial basis function · 1D-Integrated Radial Basis Function-based approximation $\cdot$ Multiscale macro-micro stochastic simulation $\cdot$ Non-dilute fibre suspension

\section{Introduction}

The regime of a suspension flow is based on two parameters: the aspect ratio $a_{r}$ and the volume fraction $\phi$. Concretely, a suspension can be either dilute, semi-dilute, or concentrated for $\phi a_{r}^{2}<1,1<\phi a_{r}^{2}<a_{r}$, or $\phi a_{r}^{2}>a_{r}$, respectively. In dilute suspensions, the interaction of fibres can be neglected and the fibre evolution can be captured by the Jeffery equation [1]. Meanwhile, the physical description of the evolution of suspension configurations poses a challenge due to the necessity to take into account fibre interactions for the semi-dilute and concentrated suspensions. The fibre parameters for cases ranging from semi-dilute to concentrated suspensions are presented in Table 1.

Besides the bulk properties of a flow, the orientation of fibres in the flow is also considered. At a position in the flow, the orientation of fibres is illustrated by an ellipse's

Canh-Dung Tran

canh-dung.tran@usq.edu.au

1 School of Engineering, Faculty of Health, Engineering and Sciences, University of Southern Queensland, West Street, Darling Heights, Toowoomba, QLD 4350, Australia geometry with three cases: a circle, an ellipse, or a straight line (see Fig. 1). They are corresponding to a predominant direction of fibres in parallel with the ellipse's long major axis, or all fibres aligning with the line at a position, respectively.

For non-dilute concentrated suspensions, the fibre-fibre interaction is significant. Thus, it is necessary to take into consideration this interaction and one possible way is to introduce a diffusion term into Jeffery's equation Folgar and Tucker [2]. From the literature, the simulation of a fiber suspension is basically carried out through three following steps: (i) introduce a fiber stress component into the momentum conservation equation to include dynamic effects of fibers on the bulk properties of the flow, (ii) apply an appropriate motion equation to describe the evolution of fiber particles, as stated above, the Jeffery's equation is suitable for dilute suspension, whereas the Folgar-Tucker's equation is applicable for semi-dilute and concentrated ones; and (iii) determine the fiber contribution to stress (named fiber stress tensor) using a relevant constitutive equation as a function of the fibers' orientation.

Since the fiber stress tensor is essentially calculated from the fourth-order orientation tensor $\langle\mathbf{P P P P}\rangle$, the basic 
Table 1 States of fibre suspension fluids: semi-dilute $\left(1 \leq \phi a_{r}^{2}<a_{r}\right)$ and concentrated $\left(\phi a_{r}^{2} \geq a_{r}\right)$, where $a_{r}$ is the aspect ratio of fibre and $\phi$ the volume fraction

\begin{tabular}{llll}
\hline$a_{r}$ & $\phi$ & $\phi a_{r}^{2}$ & State of fibre suspension \\
\hline 10 & 0.01 & 1 & Semi-dilute \\
10 & 0.02 & 2 & Semi-dilute \\
10 & 0.05 & 5 & Semi-dilute \\
10 & 0.08 & 8 & Semi-dilute \\
10 & 0.10 & 10 & Concentrated \\
10 & 0.12 & 12 & Concentrated \\
10 & 0.15 & 15 & Concentrated \\
10 & 0.18 & 18 & Concentrated \\
10 & 0.20 & 20 & Concentrated \\
20 & 0.01 & 4 & Semi-dilute \\
20 & 0.02 & 8 & Semi-dilute \\
20 & 0.05 & 20 & Concentrated \\
20 & 0.08 & 32 & Concentrated \\
20 & 0.10 & 40 & Concentrated \\
\hline
\end{tabular}

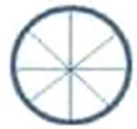

(a)

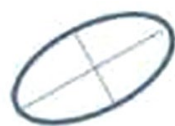

(b) (c)
Fig. 1 Orientation of fibres: a Circle: the isotropic fibres' direction; b Ellipse: the long major axis is the predominant of fibres' direction and $\mathbf{c}$ Straight line: all fibres completely align with the line

difference of numerical methods to simulate fiber suspensions is the way to handle the fourth-order tensor. There are several approaches to process the fourth-order orientation tensor. One approach is to use a quadratic closure approximation to break the tensor $\langle\mathbf{P P P P}\rangle$ into two second-order tensors $\langle\mathbf{P P}\rangle$ Lipscomb et al. [3]. A fully alignment assumption is subsequently applied to calculate these second-order tensors. This approach was employed to successfully simulate fiber suspension flows through axisymmetric contraction and expansion geometries Lipscomb et al. [3], Chiba et al. [4] and Baloch and Webster [5]. Another one is to directly solve the evolution of the fourth-order tensor as presented in Advani and Tucker [6]. And last but not least, the Brownian Configuration Field (BCF) approach Hulsen, et al. [7], Tran-Canh and Tran-Cong $[8,9]$ was successfully applied to the simulation of fiber suspensions by Fan et al. [10], $\mathrm{Lu}$ et al. [11], Dou et al. [12] and Eberle et al. [13]. Follow the approach, a high number of fiber configurational fields is initiated on each computational node and the fourth-order tensor will be averagely calculated.

Over the last decade, the macro-micro multiscale methods based on the differentiated and integrated RBF approximations have been developed to simulate successfully a range of dilute polymer solutions and melts Tran et al., [14, 15], Nguyen et al. $[16,17]$. In addition, a multiscale modelling based on the combination of IRBF, DAVSS and BCF idea has been also proposed for simulations of dilute fibre suspensions in Nguyen et al. [18]. Owing to the advantages of RBF-based high-order approximation schemes, the approach achieved high-order convergence and accuracy Nguyen et al. [16, 17].

Aligning with this approach, high-order RBF-based Brownian configuration field (BCF) method for dilute fibre suspensions Nguyen et al. [17] by our group is further developed for non-dilute suspensions by introducing a diffusion term into the Jeffery equation to capture fibre-fibre interaction. For this scheme, the conservation equations using the vorticity-stream function form are discretised using the high-order integrated RBF scheme, whereas the fibre configurations governed by the Folgar-Tucker equation are advanced by the BCF approach. Such two macro-micro processes are coupled using the PhanThien and Graham model Phan-Thien and Graham [19] for the fibre stress.

Since the present method is the combination between the Stochastic Simulation Technique and a mesh-free numerical method, it is powerful for problems with moving boundaries, complex boundary or free surface and without the need of closed form constitutive equation. Results evidenced by numerical experiments show the present method would be potential in simulating and producing suspensions in food and medical industries.

This work is organised as follows: the governing equations in the dimensionless form are first presented in Sect. 2. An introduction of the discrete adaptive viscoelastic stress splitting (DAVSS) technique into the conservation equations is also summarised in this section. Section 3 presents a coupled macro-micro system of governing equations, followed by a short review of the present method together with its algorithm. The application of the present method in semi-dilute and concentrated suspensions is then demonstrated and discussed through several benchmark examples in Sect. 4.

\section{Governing equations for non-dilute suspension flows}

The dimensionless conservation equations for fibre suspension flows are given by [11]

$\nabla \cdot \mathbf{u}=0$

$\frac{\partial \mathbf{u}}{\partial t}+\mathbf{u} \cdot \nabla \mathbf{u}=-\nabla p+\frac{1}{R e} \nabla \cdot\left(\boldsymbol{\tau}_{s}+\boldsymbol{\tau}_{f}\right)$,

where $\mathbf{u}, p$ and $t$ are the velocity, pressure and time, respectively; $\boldsymbol{\tau}_{s}=2 \mathbf{D}$ the stress contribution of the Newtonian solvent; $\mathbf{D}=\frac{1}{2}\left(\nabla \mathbf{u}+(\nabla \mathbf{u})^{T}\right)$ the rate of strain tensor; $\boldsymbol{\tau}_{f}$ the 
stress component contributed by the suspended fibres and $R e$ the Reynolds number.

In this work, the fibre stress $\boldsymbol{\tau}_{f}$ for both the semi-dilute and concentrated suspensions is determined using the modified Phan-Thien-Graham model as follows Phan-Thien and Graham [19] and Fan et al. [10]:

$\boldsymbol{\tau}_{f}=f(\phi)\left(A \mathbf{D}:\langle\mathbf{P P P P}\rangle+2 D_{r} F\langle\mathbf{P P}\rangle\right)$,

where $\langle\mathbf{P P}\rangle$ and $\langle\mathbf{P P P P}\rangle$ are the second- and the fourth-order orientation tensors, respectively, in which $\mathbf{P}$ is the unit direction vector of fibres. $D_{r}$ is the diffusion coefficient; $\langle(*)\rangle$ the average value of $(*)$; and $\mathrm{A}, \mathrm{F}$ and $f(\phi)$ are fluid parameters which are defined as functions of the volume fraction $\phi$ and the aspect ratio $a_{r}$ of the suspended fibres as follows [10]:

$A=\frac{a_{r}^{2}}{\ln 2 a_{r}-1.5}, \quad F=\frac{3 a_{r}^{2}}{\ln 2 a_{r}-0.5}, \quad f(\phi)=\frac{\phi\left(2-\phi / \phi_{m}\right)}{2\left(1-\phi / \phi_{m}\right)^{2}}$,

where $\phi_{m}$ is the maximum volume package and empirically determined as a linear function of the aspect ratio [20]:

$\phi_{m}=0.53-0.013 a_{r}, \quad 5<a_{r}<3$.

The second- and fourth-order orientation tensors, $<\mathbf{P P}>$ and $<$ PPPP $>$ in Eq. (3) are defined by

$\langle\mathbf{P P}\rangle=\frac{1}{N_{f}} \sum_{i=1}^{N_{f}} \frac{\mathbf{Q}_{i}}{Q_{i}} \frac{\mathbf{Q}_{i}}{Q_{i}}\langle\mathbf{P P P P}\rangle=\frac{1}{N_{f}} \sum_{i=1}^{N_{f}} \frac{\mathbf{Q}_{i}}{Q_{i}} \frac{\mathbf{Q}_{i}}{Q_{i}} \frac{\mathbf{Q}_{i}}{Q_{i}} \frac{\mathbf{Q}_{i}}{Q_{i}}$,

where $N_{f}$ is the number of fibre configuration fields. More details can be found in Bird et al. [21, 22].

The evolution of fibres' orientation in non-dilute suspensions is determined using the Folgar and Tucker equation Folgar and Tucker [2]:

$\frac{D \mathbf{P}}{D t}=\mathbf{L} \cdot \mathbf{P}-\mathbf{L}: \mathbf{P P P}+(\mathbf{I}-\mathbf{P P}) \cdot \mathbf{F}^{(b)}(t)$,

where $\frac{D}{D t}(\cdot)$ is the material time derivative of (.), I the identity tensor and $\mathbf{L}$ is the effective velocity gradient tensor and given by

$\mathbf{L}=(\nabla \mathbf{u})^{T}-\zeta \mathbf{D} \quad$ with $\quad \zeta=\frac{2}{a_{r}^{2}+1}$.

$$
\begin{aligned}
& \operatorname{Re}\left(\frac{\partial \omega}{\partial t}+u_{z} \frac{\partial \omega}{\partial z}+u_{r} \frac{\partial \omega}{\partial r}-\frac{u_{r}}{r} \omega\right)-\eta_{a}\left(\frac{\partial^{2} \omega}{\partial z^{2}}+\frac{\partial^{2} \omega}{\partial r^{2}}\right) \\
& =-\left(\eta_{a}-1\right)\left(\frac{\partial^{2} \omega}{\partial z^{2}}+\frac{\partial^{2} \omega}{\partial r^{2}}\right)+\left(\frac{1}{r} \frac{\partial \omega}{\partial r}-\frac{1}{r^{2}} \omega\right) \\
& \quad+\frac{1}{2}\left(\frac{\partial^{2} \tau_{f}^{r z}}{\partial r^{2}}-\frac{\partial^{2} \tau_{f}^{z r}}{\partial z^{2}}+\frac{\partial^{2} \tau_{f}^{z z}}{\partial r \partial z}-\frac{\partial^{2} \tau_{f}^{r r}}{\partial z \partial r}+\frac{1}{r} \frac{\partial \tau_{f}^{r z}}{\partial r}-\frac{1}{r} \frac{\partial \tau_{f}^{r r}}{\partial z}-\frac{1}{r^{2}} \tau_{f}^{r z}\right),
\end{aligned}
$$

It is worth noting that the fibres' interaction is random collisions by the Brownian force $\mathbf{F}^{(b)}(t)$ with the properties $\left\langle\mathbf{F}^{(b)}(t)\right\rangle=0$ and $\left\langle\mathbf{F}^{(b)}(t+s) \mathbf{F}^{(b)}(t)\right\rangle=2 D_{r} \delta(s) \mathbf{I}$, where $\delta(s)$ is the Dirac delta function; $D_{r}=C_{i} \dot{\gamma}$ the diffusion coefficient; $\dot{\gamma}=\sqrt{2(\mathbf{D}: \mathbf{D})}$ is the general strain rate and $C_{i}$ the interaction coefficient. In this work, $C_{i}$ is chosen as a constant as done in $[10,11]$ for simplicity.

By introducing $\mathbf{Q}(\mathbf{x}, t)=Q \mathbf{P}(\mathbf{x}, t)$, Eq. (7) is transformed into [23]

$\frac{\partial \mathbf{Q}}{\partial t}+\mathbf{u} \cdot \nabla \mathbf{Q}=\mathbf{L} \cdot \mathbf{Q}+\sqrt{2 C_{i} \dot{\gamma}} Q \frac{d \mathbf{W}}{d t}$,

where $Q$ is the modulus of $\mathbf{Q}$ and $\sqrt{2 C_{i} \dot{\gamma}} \frac{d \mathbf{W}}{d t}$ is the Brownian force $\mathbf{F}^{(b)}$, a function of the Wiener process $\mathbf{W}$.

\subsection{Vorticity-stream function formulation in the cylindrical coordinates}

In this work, several 2-D problems are considered using the axisymmetric vorticity-stream function in the cylindrical coordinates ( $r, z$ : radial and axial directions).

The relations between velocity $\left(u_{r}, u_{z}\right)$, and vorticity $\omega$ and stream function $\Psi$ are given by

$\omega=\frac{1}{2}\left(\frac{\partial u_{z}}{\partial r}-\frac{\partial u_{r}}{\partial z}\right), \quad u_{z}=\frac{1}{r} \frac{\partial \Psi}{\partial r} u_{r}=-\frac{1}{r} \frac{\partial \Psi}{\partial z}$.

The stream function and vorticity transport equations can be then derived from Eqs. (1) and (2) using Eq. (10), respectively, as follows:

$\frac{1}{r} \frac{\partial^{2} \Psi}{\partial z^{2}}+\frac{1}{r} \frac{\partial^{2} \Psi}{\partial r^{2}}-\frac{1}{r^{2}} \frac{\partial \Psi}{\partial r}=2 \omega$.

$$
\begin{aligned}
& R e\left(\frac{\partial \omega}{\partial t}+u_{z} \frac{\partial \omega}{\partial z}+u_{r} \frac{\partial \omega}{\partial r}-\frac{u_{r}}{r} \omega\right)=\left(\frac{\partial^{2} \omega}{\partial z^{2}}+\frac{\partial^{2} \omega}{\partial r^{2}}+\frac{1}{r} \frac{\partial \omega}{\partial r}-\frac{1}{r^{2}} \omega\right) \\
& \quad+\frac{1}{2}\left(\frac{\partial^{2} \tau_{f}^{r z}}{\partial r^{2}}-\frac{\partial^{2} \tau_{f}^{\tau r}}{\partial z^{2}}+\frac{\partial^{2} \tau_{f}^{z z}}{\partial r \partial z}-\frac{\partial^{2} \tau_{f}^{r r}}{\partial z \partial r}+\frac{1}{r} \frac{\partial \tau_{f}^{r r}}{\partial r}-\frac{1}{r} \frac{\partial \tau_{f}^{r r}}{\partial z}-\frac{1}{r^{2}} \tau_{f}^{r z}\right),
\end{aligned}
$$

where $\tau_{f}^{z z}, \tau_{f}^{z r}, \tau_{f}^{r z}$ and $\tau_{f}^{r r}$ are the components of the fibre/ suspension stress tensor $\boldsymbol{\tau}_{f}$. lows Nguyen et al. [17]:
Using the DAVSS scheme, Eq. (12) is rewritten as fol- 
where $\eta_{a}$ is the adaptive viscosity and expressed by [11]:

$\eta_{a}=A f(\phi)+\frac{1+\sqrt{(1 / 2) \boldsymbol{\tau}_{f}: \boldsymbol{\tau}_{f}}}{1+\sqrt{(1 / 2) \mathbf{G}: \mathbf{G}}}$.

With $\mathbf{G}=\nabla \mathbf{u}+(\nabla \mathbf{u})^{T}$ is twice the strain rate tensor.

\subsection{Discrete governing equations of fibre configurations in 2-D axisymmetric coordinates}

The effective velocity gradient $\mathbf{L}$ is developed in 2-D axisymmetric coordinate $(\mathrm{z}, \mathrm{r})$ as follows:

$\mathbf{L}=\left[\begin{array}{cc}(1-\zeta) \frac{\partial u_{z}}{\partial z} & \frac{\partial u_{z}}{\partial r}-\frac{\zeta}{2}\left(\frac{\partial u_{z}}{\partial r}+\frac{\partial u_{r}}{\partial z}\right) \\ \frac{\partial u_{r}}{\partial z}-\frac{\zeta}{2}\left(\frac{\partial u_{z}}{\partial r}+\frac{\partial u_{r}}{\partial z}\right) & (1-\zeta) \frac{\partial u_{r}}{\partial r}\end{array}\right]$.

With Eq. (15), the evolution equation(9) is developed in $z$ and $r$-directions as

$$
\begin{aligned}
\tau_{f}^{z r}=\tau_{f}^{r z}= & f(\phi) A\left[\frac{\partial u_{z}}{\partial z}\left\langle P_{z} P_{z} P_{z} P_{r}\right\rangle\right. \\
& +\left(\frac{\partial u_{z}}{\partial r}+\frac{\partial u_{r}}{\partial z}\right)\left\langle P_{z} P_{z} P_{r} P_{r}\right\rangle \\
& \left.+\frac{\partial u_{r}}{\partial r}\left\langle P_{z} P_{r} P_{r} P_{r}\right\rangle\right] \\
& +2 f(\phi) D_{r} F\left\langle P_{z} P_{r}\right\rangle
\end{aligned}
$$

\section{Numerical method of the present method for non-dilute fibre suspension flows}

A macro-micro multiscale system included Eqs. (11), (13), (16a \& b) and (17a, b \& c) for the governing equations together with the stress formula in 2-D axisymmetric coordinates is discretised using the high-order radial basis

$\frac{\partial Q_{z}}{\partial t}+u_{z} \frac{\partial Q_{z}}{\partial z}+u_{r} \frac{\partial Q_{z}}{\partial r}=(1-\zeta) \frac{\partial u_{z}}{\partial z} Q_{z}+\left[\frac{\partial u_{z}}{\partial r}-\frac{\zeta}{2}\left(\frac{\partial u_{z}}{\partial r}+\frac{\partial u_{r}}{\partial z}\right)\right] Q_{r}+\sqrt{2 C_{i} \dot{\gamma}} Q d W_{z}$,

$\frac{\partial Q_{r}}{\partial t}+u_{z} \frac{\partial Q_{r}}{\partial z}+u_{r} \frac{\partial Q_{r}}{\partial r}=(1-\zeta) \frac{\partial u_{r}}{\partial r} Q_{r}+\left[\frac{\partial u_{r}}{\partial z}-\frac{\zeta}{2}\left(\frac{\partial u_{z}}{\partial r}+\frac{\partial u_{r}}{\partial z}\right)\right] Q_{z}+\sqrt{2 C_{i} \dot{\gamma}} Q d W_{r}$.

Using tensor products, the fibre stress tensor in Eq. (3) can be developed in the $\mathrm{z}$ - and $\mathrm{r}$-coordinates as follows: function-based BCF method Nguyen et al. [17] as follows.

The vorticity equation (13) is temporally discretised using the semi-implicit scheme as follows:

$$
\begin{aligned}
\omega^{n+1}-\frac{\Delta t}{\operatorname{Re}} \eta_{a}\left(\frac{\partial^{2} \omega^{n+1}}{\partial z^{2}}+\frac{\partial^{2} \omega^{n+1}}{\partial r^{2}}\right)= & \omega^{n}-\frac{\Delta t}{\operatorname{Re}}\left(\eta_{a}-1\right)\left(\frac{\partial^{2} \omega^{n}}{\partial z^{2}}+\frac{\partial^{2} \omega^{n}}{\partial r^{2}}\right) \\
& +\frac{\Delta t}{\operatorname{Re}}\left(\frac{1}{r} \frac{\partial \omega^{n}}{\partial r}-\frac{1}{r^{2}} \omega^{n}\right)-\Delta t\left(u_{z}^{n} \frac{\partial \omega^{n}}{\partial z}-u_{r}^{n} \frac{\partial \omega^{n}}{\partial r}+\frac{u_{r}^{n}}{r} \omega^{n}\right) \\
& +\frac{\Delta t}{2 \operatorname{Re}}\left(\frac{\partial^{2}\left(\tau_{f}^{r z}\right)^{n}}{\partial r^{2}}-\frac{\partial^{2}\left(\tau_{f}^{z r}\right)^{n}}{\partial z^{2}}+\frac{\partial^{2}\left(\tau_{f}^{z z}\right)^{n}}{\partial r \partial z}-\frac{\partial^{2}\left(\tau_{f}^{r r}\right)^{n}}{\partial z \partial r}+\frac{1}{r} \frac{\partial\left(\tau_{f}^{r z}\right)^{n}}{\partial r}\right. \\
& \left.-\frac{1}{r} \frac{\partial\left(\tau_{f}^{r r}\right)^{n}}{\partial z}-\frac{1}{r^{2}}\left(\tau_{f}^{r z}\right)^{n}\right) .
\end{aligned}
$$

$$
\begin{aligned}
\tau_{f}^{z z}= & f(\phi) A\left[\frac{\partial u_{z}}{\partial z}\left\langle P_{z} P_{z} P_{z} P_{z}\right\rangle+\left(\frac{\partial u_{z}}{\partial r}+\frac{\partial u_{r}}{\partial z}\right)\left\langle P_{z} P_{z} P_{z} P_{r}\right\rangle+\frac{\partial u_{r}}{\partial r}\left\langle P_{z} P_{z} P_{r} P_{r}\right\rangle\right] \\
& +2 f(\phi) D_{r} F\left\langle P_{z} P_{z}\right\rangle,
\end{aligned}
$$$$
\tau_{f}^{r r}=f(\phi) A\left[\frac{\partial u_{z}}{\partial z}\left\langle P_{z} P_{z} P_{r} P_{r}\right\rangle+\left(\frac{\partial u_{z}}{\partial r}+\frac{\partial u_{r}}{\partial z}\right)\left\langle P_{z} P_{r} P_{r} P_{r}\right\rangle+\frac{\partial u_{r}}{\partial r}\left\langle P_{r} P_{r} P_{r} P_{r}\right\rangle\right]
$$$$
+2 f(\phi) D_{r} F\left\langle P_{r} P_{r}\right\rangle
$$ 
For the microscale, the evolution Eq. (16a \& b) are explicitly discretised using the Euler-Maruyama method:

$$
\begin{aligned}
Q_{z}^{n+1}= & Q_{z}^{n}-\left(u_{z} \frac{\partial Q_{z}^{n}}{\partial z}+u_{r} \frac{\partial Q_{z}^{n}}{\partial r}\right) \Delta t+(1-\zeta) \frac{\partial u_{z}}{\partial z} Q_{z}^{n} \Delta t \\
& +\left[\frac{\partial u_{z}}{\partial r}-\frac{\zeta}{2}\left(\frac{\partial u_{z}}{\partial r}+\frac{\partial u_{r}}{\partial z}\right)\right] Q_{r}^{n} \Delta t+\sqrt{2 C_{i} \dot{\gamma} \Delta t} Q W_{z}^{n}, \\
Q_{r}^{n+1}= & Q_{r}^{n}-\left(u_{z} \frac{\partial Q_{r}^{n}}{\partial z}+u_{r} \frac{\partial Q_{r}^{n}}{\partial r}\right) \Delta t+(1-\zeta) \frac{\partial u_{r}}{\partial r} Q_{r}^{n} \Delta t \\
& +\left[\frac{\partial u_{r}}{\partial z}-\frac{\zeta}{2}\left(\frac{\partial u_{z}}{\partial r}+\frac{\partial u_{r}}{\partial z}\right)\right] Q_{z}^{n} \Delta t+\sqrt{2 C_{i} \dot{\gamma} \Delta t} Q W_{r}^{n},
\end{aligned}
$$

where superscripts $(n+1)$ and $n$ denote the two successive time steps at $t^{n+1}=(n+1) \Delta t$ and $t^{n}=n \Delta t$, respectively; $\Delta t$ is the time step size.

At each time step, the vorticity and/or stream function equations, as well as the first and second derivatives of the field variables including the fibre stresses at the collocation points, are approximated using the 1-D integrated RBF (1D-IRBF) method which is presented in Sect. 3.1.

\subsection{The 1D-IRBF-based spatial discretisation scheme}

At a time $\mathrm{t}$, the highest order derivative of dependent variable $\omega(x, t)$ (the second order in this work), the first-order derivatives and the function itself are decomposed as follows MaiDuy et al. [24] and Thai-Quang et al. [25]:

$\frac{d^{2} \omega}{d x^{2}}=\sum_{j=1}^{m} w_{j}(t) g_{j}(x)=\sum_{j=1}^{m} w_{j}(t) G_{j}^{[2]}(x)$, where $\left\{w_{j}(\mathrm{t})\right\}_{j=1}^{m}$ is the RBF weights; $\left\{g_{j}(\mathrm{x})\right\}_{j=1}^{m}$ the RBFs; m a chosen number; $G_{j}^{[1]}(x)=\int G_{j}^{[2]}(x) d x$; $G_{j}^{[0]}(x)=\int G_{j}^{[1]}(x) d x$ and $C_{1}$ and $C_{2}$ are unknown integration constants at time $t$. In this work, the multi-quadric RBF (MQRBF) is used and given by

$g_{j}(x)=\sqrt{\left(x-c_{j}\right)+a_{j}^{2}}$,

where $\left\{c_{j}\right\}_{j=1}^{m}$ and $\left\{a_{j}\right\}_{j=1}^{m}$ are the RBF centres and widths, respectively. The centres are chosen to be the same as the data points $x_{j}$ in this work.

Equations (20a), (20b) and (20c) are evaluated at each and every collocation point and then rearranged to produce the following a set of algebraic equations:

$\frac{d^{2} \boldsymbol{\omega}}{d x^{2}}=\hat{\mathbf{G}}^{[2]}(x) \hat{\mathbf{w}}(t)$,

$\frac{d \boldsymbol{\omega}}{d x}=\hat{\mathbf{G}}^{[1]}(x) \hat{\mathbf{w}}(t)$,

$\boldsymbol{\omega}=\hat{\mathbf{G}}^{[0]}(x) \hat{\mathbf{w}}(t)$,

where

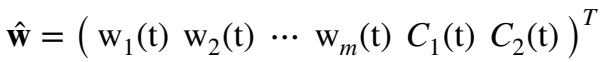

$$
\begin{aligned}
& \hat{\boldsymbol{\omega}}=\left(\begin{array}{llll}
\omega_{1}(t) & \omega_{2}(t) & \cdots & \omega_{m}(t)
\end{array}\right)^{T} \quad \text { with } \omega_{j}=\omega\left(x_{j}\right),
\end{aligned}
$$

$\frac{d^{k} \hat{\boldsymbol{\omega}}}{d \mathrm{x}^{k}}=\left(\frac{d^{k} \omega_{1}(\mathrm{x}, \mathrm{t})}{d \mathrm{x}^{k}} \frac{d^{k} \omega_{2}(\mathrm{x}, \mathrm{t})}{d \mathrm{x}^{k}} \cdots \frac{d^{k} \omega_{m}(\mathrm{x}, \mathrm{t})}{d \mathrm{x}^{k}}\right)^{T} \quad$ with $\mathrm{k}=\{1,2\}$,

$$
\begin{aligned}
\hat{\mathbf{G}}^{[2]} & =\left[\begin{array}{ccccc}
G_{1}^{[2]}\left(\mathrm{x}_{1}\right) & \cdots & G_{m}^{[2]}\left(\mathrm{x}_{1}\right) & 0 & 0 \\
G_{1}^{[2]}\left(\mathrm{x}_{2}\right) & \cdots & G_{m}^{[2]}\left(\mathrm{x}_{2}\right) & 0 & 0 \\
\vdots & \ddots & \vdots & \vdots & \vdots \\
G_{1}^{[2]}\left(\mathrm{x}_{m}\right) & \cdots & G_{m}^{[2]}\left(\mathrm{x}_{m}\right) & 0 & 0
\end{array}\right] \quad \hat{\mathbf{G}}^{[1]}=\left[\begin{array}{ccccc}
G_{1}^{[1]}\left(\mathrm{x}_{1}\right) & \cdots & G_{m}^{[1]}\left(\mathrm{x}_{1}\right) & 1 & 0 \\
G_{1}^{[1]}\left(\mathrm{x}_{2}\right) & \cdots & G_{m}^{[1]}\left(\mathrm{x}_{2}\right) & 1 & 0 \\
\vdots & \ddots & \vdots & \vdots & \vdots \\
G_{1}^{[1]}\left(\mathrm{x}_{m}\right) & \cdots & G_{m}^{[1]}\left(\mathrm{x}_{m}\right) & 1 & 0
\end{array}\right] \\
\hat{\mathbf{G}}^{[0]} & =\left[\begin{array}{ccccc}
G_{1}^{[0]}\left(\mathrm{x}_{1}\right) & \cdots & G_{m}^{[0]}\left(\mathrm{x}_{1}\right) & \mathrm{x}_{1} & 1 \\
G_{1}^{[0]}\left(\mathrm{x}_{2}\right) & \cdots & G_{m}^{[0]}\left(\mathrm{x}_{2}\right) & \mathrm{x}_{2} & 1 \\
\vdots & \ddots & \vdots & \vdots & \vdots \\
G_{1}^{[0]}\left(\mathrm{x}_{m}\right) & \cdots & G_{m}^{[0]}\left(\mathrm{x}_{m}\right) & \mathrm{x}_{m} & 1
\end{array}\right] .
\end{aligned}
$$

$\frac{d \omega}{d x}=\sum_{j=1}^{m} w_{j}(t) G_{j}^{[1]}(x)+C_{1}(t)$

$\omega(x, t)=\sum_{j=1}^{m} w_{j}(t) G_{j}^{[0]}(x)+C_{1}(t) x+C_{2}(t)$,
Owing to the presence of integration constants, more additional constraints can be incorporated into the algebraic equation system through Eq. (22c) as follows:

$\left(\begin{array}{c}\hat{\boldsymbol{\omega}} \\ \hat{\mathbf{f}}\end{array}\right)=\hat{\mathbf{C}} \hat{\mathbf{w}}$, 
where $\hat{\mathbf{C}}=\left[\begin{array}{c}\hat{\mathbf{G}}^{[0]} \\ \hat{\mathbf{L}}\end{array}\right]$ and $\hat{\mathbf{f}}=\hat{\mathbf{L}} \hat{\mathbf{w}}$ are additional constraints. The conversion of the network-weight space into the physical space yields

$\hat{\mathbf{w}}=\hat{\mathbf{C}}^{-1}\left(\begin{array}{c}\hat{\boldsymbol{\omega}} \\ \hat{\mathbf{f}}\end{array}\right)$

where $\hat{\mathbf{C}}^{-1}$ is the conversion matrix. Equation (24) is substituted into Eq. (20a) and (20b) to obtain the second- and first-order derivatives of $\omega$ in terms of nodal variable values as follows:

$\frac{d^{2} \omega}{d x^{2}}=D_{2} \hat{\boldsymbol{\omega}}+k_{2} \quad \frac{d \omega}{d x}=D_{1} \hat{\boldsymbol{\omega}}+k_{1}$,

where $D_{1}$ and $D_{2}$ are known vectors of length $\mathrm{m}$; and $k_{2}$ and $k_{1}$ are scalars determined by $\hat{\mathbf{f}}$. Apply Eq. (25) at all collocation points on the grid lines, we have

$\frac{d^{2} \boldsymbol{\omega}}{d x^{2}}=\hat{D}_{2 x} \hat{\boldsymbol{\omega}}+\hat{\mathbf{k}}_{2 x} \quad \frac{d \boldsymbol{\omega}}{d x}=\hat{D}_{1 x} \hat{\boldsymbol{\omega}}+\hat{\mathbf{k}}_{1 x}$,

where $\hat{D}_{2 x}$ and $\hat{D}_{1 x}$ are known matrices of dimension $m \times m$; and $\hat{\boldsymbol{\kappa}}_{2 x}, \hat{\boldsymbol{\kappa}}_{1 x}$ are known vectors of length $\mathrm{m} ; \mathrm{m}$ is defined as before. The subscript $x$ expresses the spatial direction, in which the matrices $\hat{D}_{2 x}, \hat{D}_{1 x}$ and the vectors $\hat{\mathbf{\kappa}}_{2 x}, \hat{\mathbf{\kappa}}_{1 x}$ are constructed. For 2-D problems, a similar process is carried out in the $y$-direction to achieve known matrices and vectors $\hat{D}_{2 y}, \hat{D}_{1 y}, \hat{\boldsymbol{\kappa}}_{2 y}$ and $\hat{\mathbf{\kappa}}_{1 y}$.

\subsection{Algorithm of the present macro-micro multiscale method}

The algorithm of the present multiscale method is detailed as follows.

- The fibre stresses are determined at a time step $t^{i+1}$ from the fibre configuration at step $t^{i}$ (the initial one for the first step) using Eq. (17a, b \& c), and the derivatives of stresses are then approximated using the high-order integral RBF technique;

- Solve Eqs. (18) and (11) for the vorticity and stream function, respectively. Then, calculate the velocity of the current step using Eq. (10);

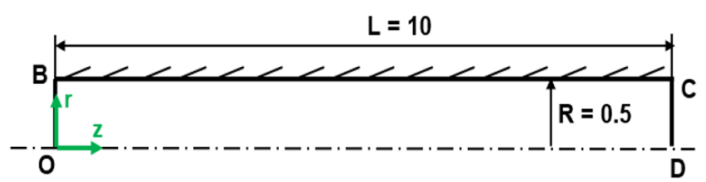

Fig. 2 Non-dilute fibre suspension flow through a circular tube: the geometry and the selected coordinates $(z, r)$
- Calculate the effective velocity gradient $\mathbf{L}$ using Eq. (15);

- Solve Eq. (19a \& b) for the fibre configuration fields Q's using the Euler-Maruyama method;

- Calculate the fibre stress tensor at step $t^{i+1}$ use Eq. (17a, $\mathrm{b} \& \mathrm{c})$;

- The routine is repeated until either the desired time or convergence measure $(\mathrm{CM})$ for the velocity is reached.

\section{Numerical examples}

The present method is used to simulate two challenging nondilute fibre suspension flows: flows through 4:1 and 4.5:1 axisymmetric contractions, and flows through 1:4 axisymmetric expansion for several fibre parameters and Reynolds numbers. The fibre parameters for cases ranging from semidilute to concentrated suspensions are presented in Table 1 of the introduction.

\subsection{Flow through a circular tube}

Although the flow through a circular tube of non-dilute fibre suspensions is not a focus of this work, this numerical example is the first investigation, because the velocity profile, the vorticity and stream function at the outlet by the simulation will be used as the boundary conditions at the inlet of the fibre suspension flow through an axisymmetric contraction and expansion presented in Sect. 4.2.

The flow through a circular tube is described in Fig. 2. The length and radius of the tube are $L=10$ and $R=0.5$, respectively. This simulation is carried out with $R e=0$, $\Delta t=1 E-3$ and the number of fibres $N_{f}=1000$.

The boundary conditions are given as follows: (i) non-slip boundary condition on the wall $\mathrm{BC}: u_{z}=0$ and $u_{r}=0$; (ii)

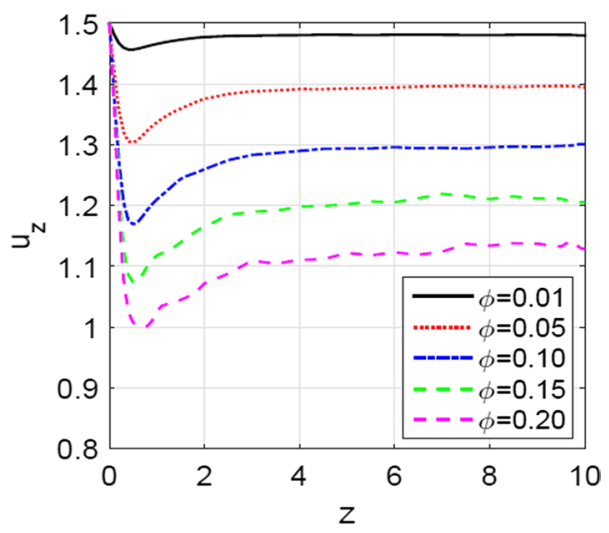

Fig. 3 Non-dilute fibre suspension flow through a circular tube: the velocity profile $\left(u_{z}\right)$ along the centreline of flows with a range of $\phi a_{r}^{2}=\{1,5,10,15,20\}$ 
Fig. 4 Non-dilute fibre suspension flow through a circular tube: the effect of fibre parameters on the velocity profile along the fibre direction with $\mathbf{a} \phi a_{r}^{2}=1 ; \mathbf{b} \phi a_{r}^{2}=10 ;$ and $\mathbf{c}$ $\phi a_{r}^{2}=20$
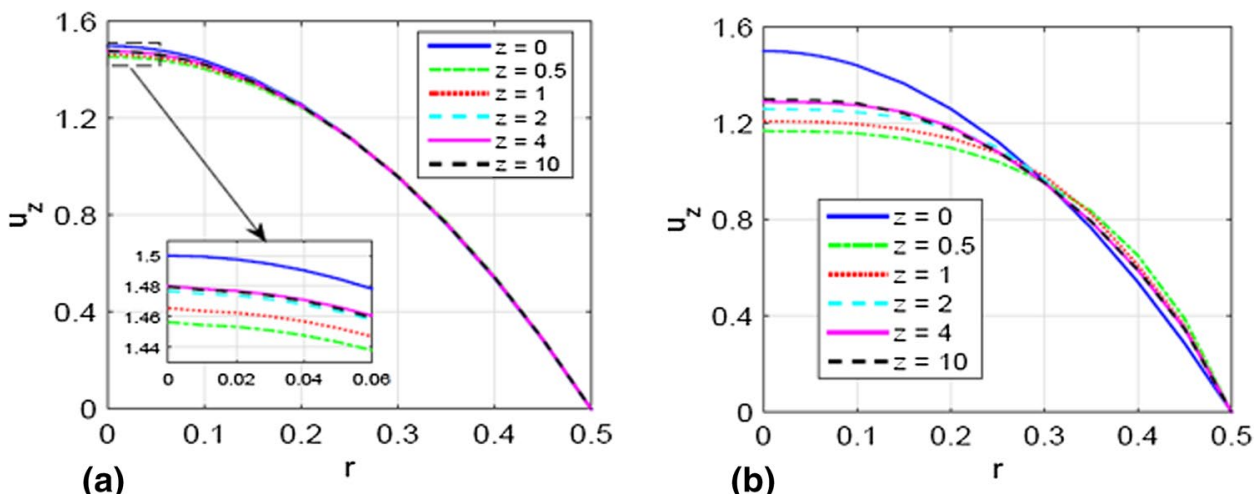

(b)

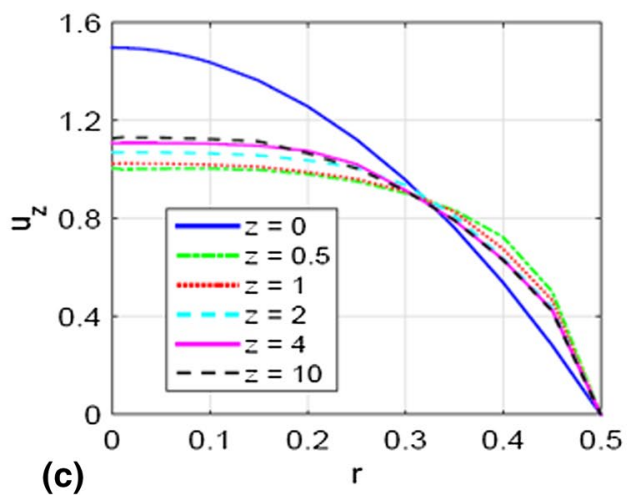

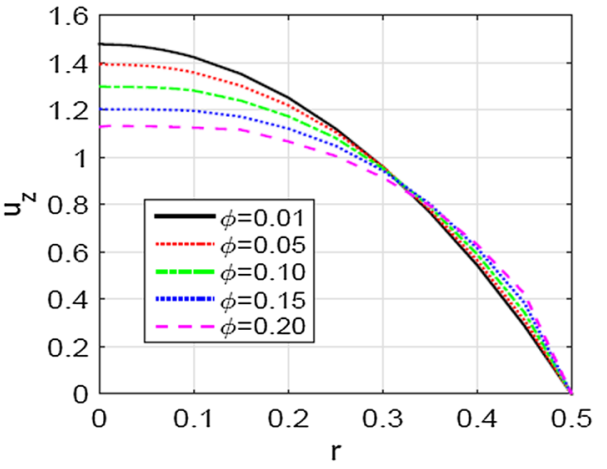

Fig. 5 Non-dilute fibre suspension flow through a circular tube: the velocity profiles at the outlet of the channel for flows with $\phi a_{r}^{2}=\{1,5,10,15,20\}$

the symmetric boundary condition on the centreline OD: $\frac{\partial u_{z}}{\partial r}=0$ and $u_{r}=0$; (iii) Newtonian parabolic velocity profile at the inlet OB: $u_{z}=u_{\max }\left(1-\left(\frac{r}{R}\right)^{2}\right)$ and $u_{r}=0$, where $u_{\max }=1.5$ is the maximum velocity. A set of $N_{f}$ fibres is randomly generated and assigned at each collocation point as the initial fibre configuration field; and (iv) flow out condition at the outlet DC: $u_{r}=0$ and $\frac{\partial u_{z}}{\partial z}=0$.

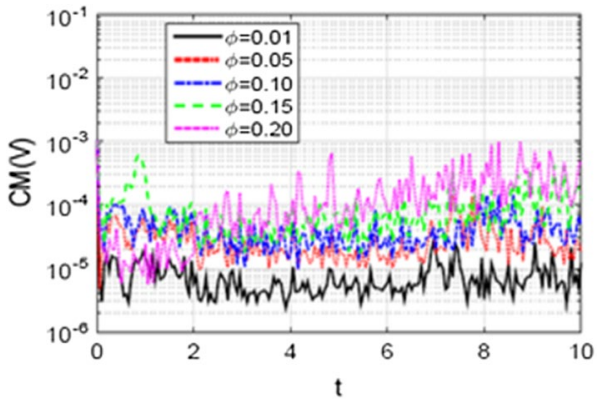

Fig. 6 Non-dilute fibre suspension flow through a circular tube: the convergence measure for the velocity of flows with $\phi a_{r}^{2}=\{1,5,10,15,20\}$

Experiences find that finer meshes near the centreline and the outlet are necessary for higher solution accuracy in these regions. Therefore, a designed grid is applied with $\Delta z_{1}=0.05, \forall z \in[0,9.8]$ and $\Delta z_{2}=0.01, \forall z \in[9.8,10]$; $\Delta r_{1}=0.01, \forall r \in[0,0.1] ;$ and $\Delta r_{2}=0.05, \forall r \in[0.1,0.5]$.

To demonstrate the role of fibre parameters in the kinetic behaviour of the flow, the non-dilute fibre suspension flow is simulated for a range of $\phi a_{r}^{2}$. The obtained results by the 


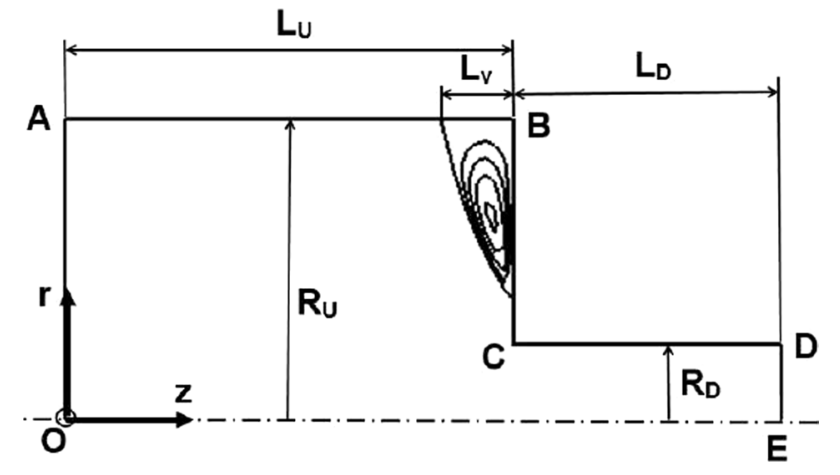

Fig. 7 Geometry of the axisymmetric contraction flow and the selected coordinates $(z, r)$

present method are discussed and compared with others as follows.

- Figure 3 presents the distribution of the axial velocity $u_{z}$ along the centreline of the tube for a range of $\phi a_{r}^{2}=\{1,5,8,10,15,20\}$. Their corresponding volume fractions $(\phi)$ and aspect ratios $\left(a_{r}\right)$ can be found in Table 1. Results show that in all cases of $\phi a_{r}^{2}$, undershoot is observed near the entrance $(z \in(0.41,0.74))$. Moreover, the undershoot is more pronounced with increasing $\phi a_{r}^{2}$. Undershoots reflect the effect of the isotropic configuration of fibres (see Fig. 1a) at the inlet. In fact, the isotropy of fibre configurations resists the development of the velocity $\left(u_{z}\right)$ at the region near the inlet. The velocity then increases along the centreline towards the outlet with a gradual decrease of the isotropy of fibres' orientation.

Results in Fig. 3 also show that the velocity $\left(u_{z}\right)$ on the centreline at the steady state reduces as $\phi$ and/or $a_{r}$ increases.

- Figure 4 shows the impact of $\phi$ and $a_{r}$ on the profile of axial velocity $\left(u_{z}\right)$ at several cross sections ( $\mathrm{z}=\{0,0.5,1,2,4,10\})$ with $\phi a_{r}^{2}=1$ (semi-dilute) (Fig. 4a), $\phi a_{r}^{2}=10$ (concentrated) (Fig. 4b) and $\phi a_{r}^{2}=20$ (concentrated) (Fig. 4c). Numerical results show that the

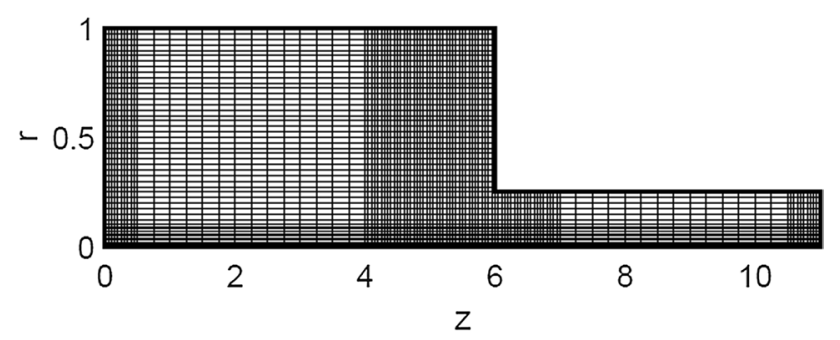

Fig. 8 Non-uniform Cartesian grid for the 4:1 axisymmetric contraction flow velocity profile becomes more plug-like with increasing $\phi$ and/or $a_{r}$. The profiles of outlet velocity of flows for a range of $\phi a_{r}^{2}=\{1,5,10,15,20\}$ presented in Fig. 5 also depict that the velocity profiles are more plug-like for higher values of $a_{r}$ and/or $\phi$.

- Finally, the influence of $\phi a_{r}^{2}$ on the convergence measure CM for the velocity is reported in Fig. 6. Results show a significant influence of $\phi a_{r}^{2}$ on the convergence of the method, where the CM generally degenerates with increasing level of fibre concentration and/or aspect ratio.

\subsection{The 4:1 axisymmetric contraction flows}

The geometry of the 4:1 axisymmetric contraction flow is schematically described in Fig. 7 with the length and radius of the upstream tube $L_{U}$ and $R_{U}$, respectively; the length and radius of the downstream $L_{D}$ and $R_{D}$; and the length of the salient vortex $L_{v}$.

The contraction ratio $(\beta)$ and the dimensionless vortex length $\left(L_{v}^{*}\right)$ are given by

$\beta=\frac{R_{U}}{R_{D}}, \quad L_{v}^{*}=\frac{L_{v}}{2 R_{U}}$.

The boundary conditions for this problem are as follows:

(i) At the inlet $\overline{O A}$, the velocity, the vorticity and the stream function are the values at the outlet of the circular Poiseuille flows investigated in Sect. 4.1. Furthermore, $\frac{\partial \Psi}{\partial z}=0$ is also assigned;

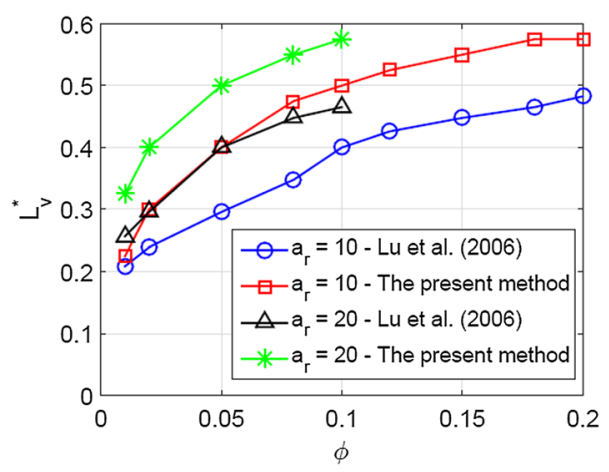

Fig. 9 4:1 axisymmetric contraction of non-dilute fibre suspensions: the impact of fibre parameters on the vortex length $\left(L_{v}^{*}\right)$ with $a_{r}=10$ and a range of $\phi \in\{0.01,0.02,0.05,0.08,0.10,0.12,0.15,0.18,0.20\} ;$ and $a_{r}=20$ and a range of $\phi \in\{0.01,0.02,0.05,0.08,0.10\}$ 
Fig. 10 4:1 axisymmetric contraction flows of non-dilute fibre suspensions: the effect of $\phi a_{r}^{2}$ on the salient corner vortex size for $\phi a_{r}^{2}=\{5,10,15,20\}$

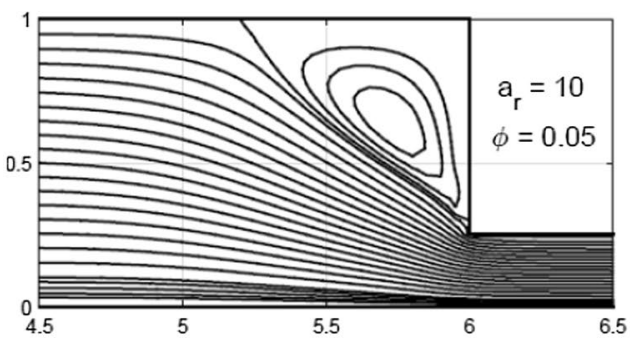

(a)

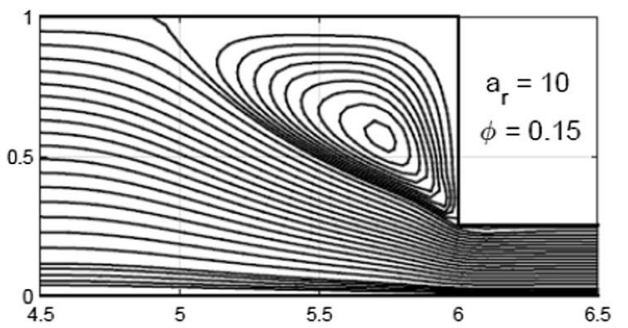

(c)

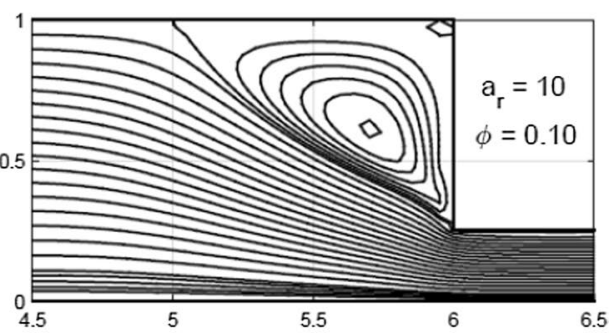

(b)

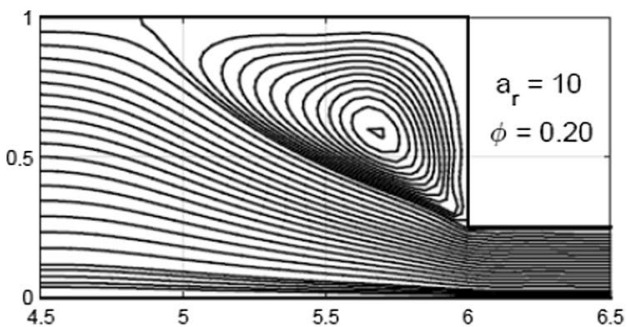

(d)

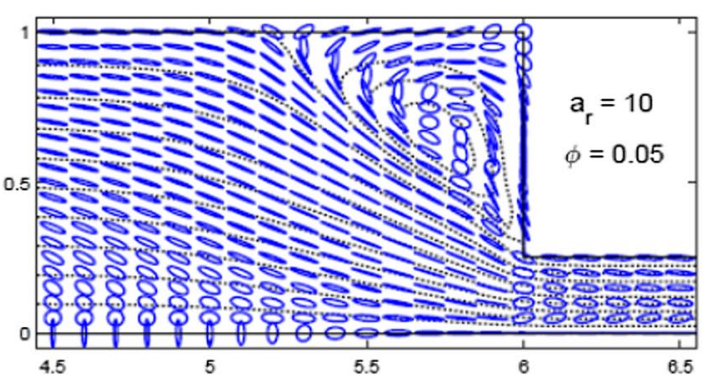

(a)

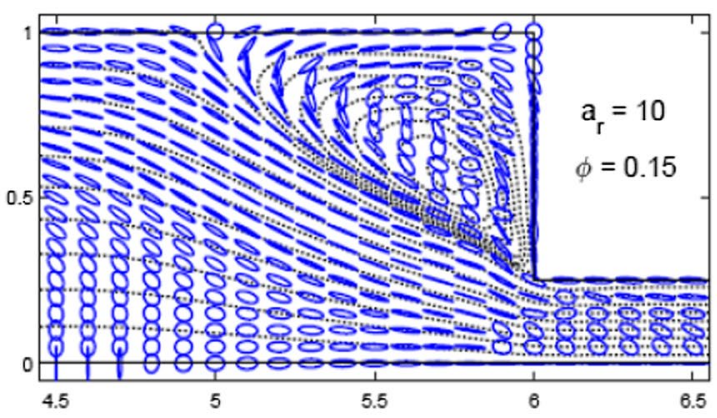

(c)

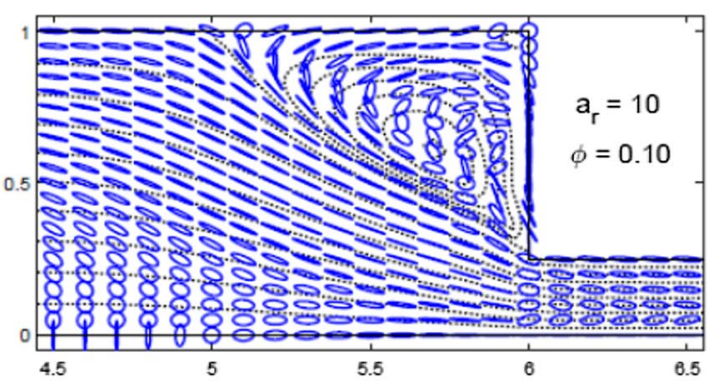

(b)

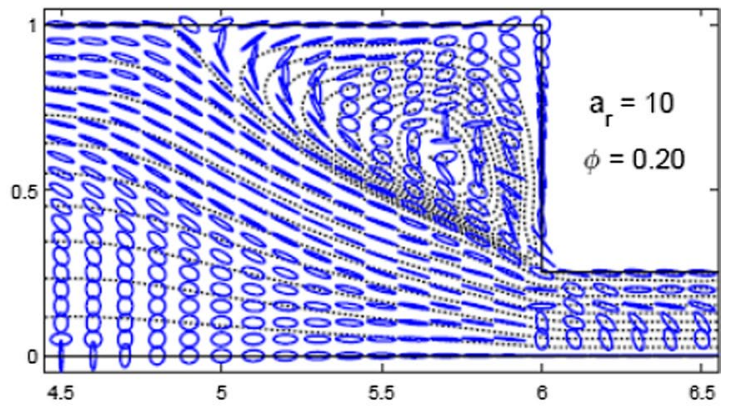

(d)

Fig. $114: 1$ axisymmetric contraction flows of non-dilute fibre suspensions: the effect of $\phi a_{r}^{2}$ on the orientation of fibres around the contraction area for $\phi a_{r}^{2}=\{5,10,15,20\}$

(ii) At the outlet $\overline{D E}$, flow-out conditions are defined as $\frac{\partial u_{z}}{\partial z}=0, u_{r}=0 ; \frac{\partial \Psi}{\partial z}=0 ;$ and $\frac{\partial \omega}{\partial z}=0$;

(iii) On the walls $\overline{A B}, \overline{C D}$ and $\frac{\partial z}{B C}$, non-slip boundary condition is set up for the velocity, i.e., $u_{z}=0$ and $u_{r}=0$. The corresponding boundary conditions for the stream function and the vorticity are determined by
- On the wall $\overline{A B}: \Psi=0, \frac{\partial \Psi}{\partial \psi}=0 ; \omega=\omega_{w}$;

- On the wall $\overline{B C}: \Psi=0, \frac{\partial \psi}{\partial z}=0 ; \omega=\omega_{w_{2}}$;

- On the wall $\overline{C D}: \Psi=0, \frac{\partial \Psi}{\partial r}=0 ; \omega=\omega_{w_{3}}$; where $\omega_{w_{1}}$, $\omega_{w_{2}}$ and $\omega_{w_{3}}$ are determined and updated using Eq. (11) with the known stream function at each time step;

(iv) On the centreline $\overline{O E}$, symmetric boundary condition for the velocity, i.e., $\frac{\partial \mathrm{u}_{z}}{\partial \mathrm{r}}=0$ and $\mathrm{u}_{r}=0$; the 
corresponding boundary conditions for the stream function and vorticity are $\Psi=\Psi_{c}, \frac{\partial \Psi}{\partial \mathrm{r}}=0$ and $\omega=0$, where $\Psi_{c}$ is determined by Eq. (10).

The non-uniform grid designed in [17] is applied to the simulation of this example as follows: $\Delta z_{1}=0.05, \forall z \in[0,0.5] \bigcup[4,7] \bigcup[10.5,11] ; \quad \Delta z_{2}=0.1$ $\forall z \in[0.5,4] \bigcup[7,10.5] ; \quad \Delta r_{1}=0.01 \quad \forall r \in[0,0.1] \quad$ and $\Delta r_{2}=0.025 \forall r \in[0.1,1]$ (see Fig. 8).

Number of fibres $N_{f}=1000$ and the time step size $\Delta t=1 E-3$ are applied in this simulation. However, a finer time step is used at the initial time for the numerical stability of the method in several cases of highly concentrated suspensions. In addition, unless otherwise stated, the simulation is carried out for the 4:1 contraction flow with $L_{U}=6$ , $R_{U}=1, L_{D}=5, R_{D}=0.25$ and $R e=0$.

The effect of the volume fraction $(\phi)$ and the fibre aspect ratio $\left(a_{r}\right)$ on the flow pattern and fibres' orientation is first investigated. Results by the present method are discussed and compared with those by [11] using the BCF-finite-element method as follows.

- Figure 9 depicts the effect of the fibre parameters $\left(\phi, a_{r}\right)$ on the vortex length $\left(L_{v}^{*}\right)$ for two cases of fibre suspension: $a_{r}=10$ with a range of $\phi \in\{0.01,0.02,0.05,0.08,0.10,0.12,0.15,0.18,0.20\}$

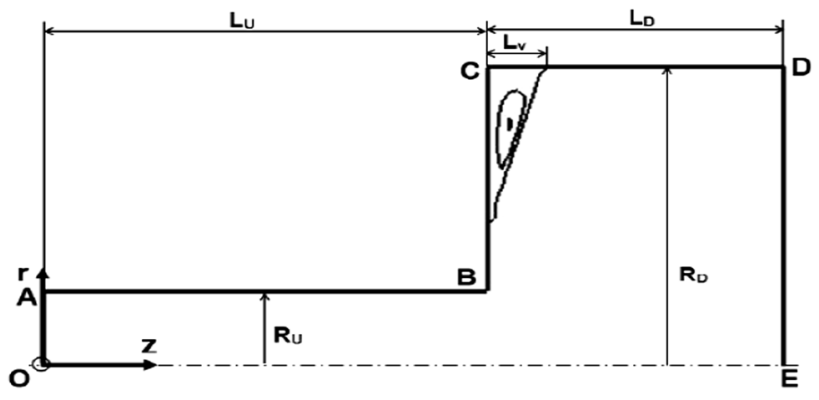

Fig. 13 Geometry for the 1:4 axisymmetric expansion flow and the selected coordinates $(z, r)$

and $\quad a_{r}=20 \quad$ with a range of $\phi \in\{0.01,0.02,0.05,0.08,0.10\}$. Numerical experiments show that the vortex length is more pronounced with the increase of $\phi$ or/and $a_{r}$. This observation was reported in [11] but with a gap in the results by the present method as shown in Fig. 9.

- The impact of fibre parameters on the shape and length of the cortex at the salient corner for a range of $\phi a_{r}^{2}=\{5,10,15,20\}$ is presented in Fig. 10 in which the salient vortex's size is more pronounced with increasing $\phi a_{r}^{2}$. Furthermore, the present results also confirm that unlike the Newtonian flow, where the vortex boundary is

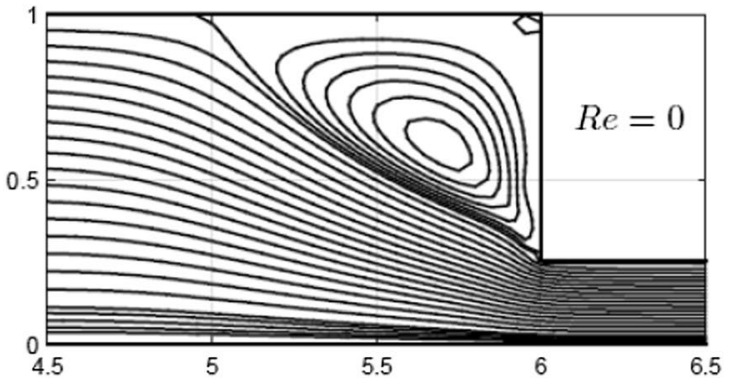

(a)

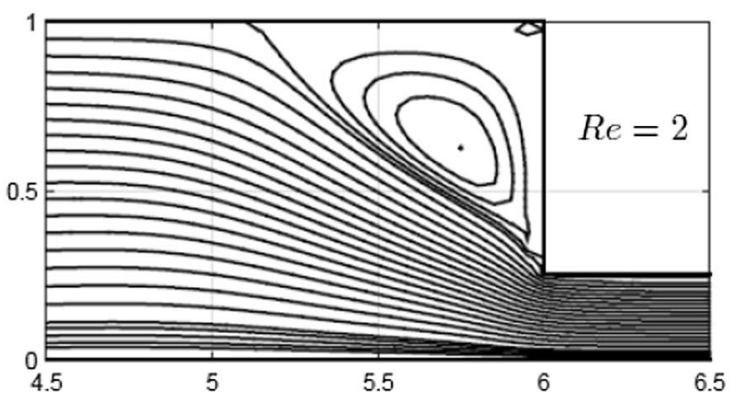

(c)

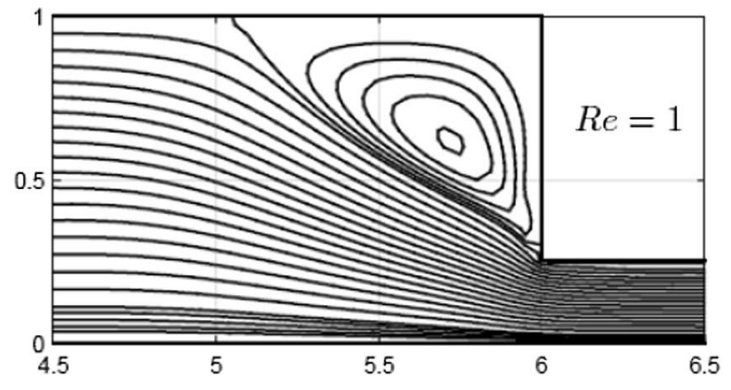

(b)

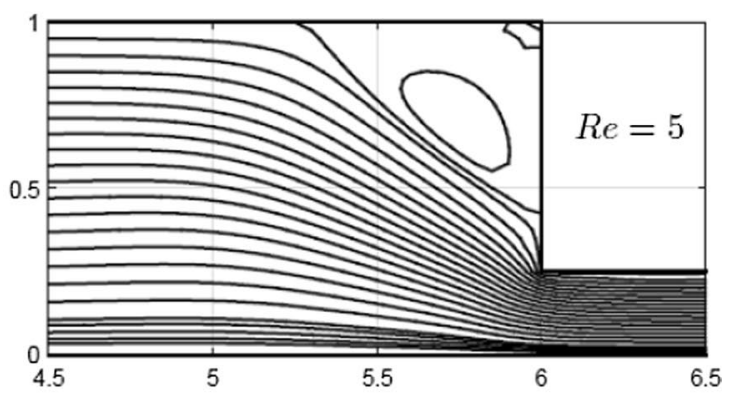

(d)

Fig. 12 4:1 axisymmetric contraction of non-dilute suspensions: the effect of Reynold number $(R e)$ on the salient corner for $\phi a_{r}^{2}=12$ 


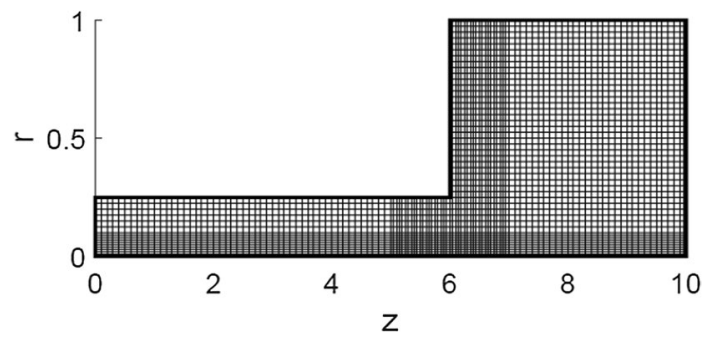

Fig. 14 Non-uniform grid for the 1:4 axisymmetric expansion flow

concave, it is slightly convex for fibre suspension flows as reported in Lipscomb et al. [3], Chiba et al. [4] and Lu et al. [11].

- Figure 11 clearly shows the influence of $\phi a_{r}^{2}$ on the orientation of fibres around the abrupt contraction by an ellipse/line/circle (as defined in Fig. 1) at a position, whereas the black dashed lines represent the streamlines for four cases of $\phi a_{r}^{2}=\{5,10,15,20\}$. Several notable points are observed as follows.

- Fibre distribution in the main area of the flow tends to be less anisotropic with the increasing of $\phi a_{r}^{2}$. Experimental results also depict that the region dominated by anisotropic fibres distribution is gradually shrinking from lower $\phi a_{r}^{2}=5$ for semidilute (Fig. 11a) to higher $\phi a_{r}^{2}=20$ for concentrated (Fig. 11d). This can be explained by the random interaction between fibres, which is stronger with increasing $\phi a_{r}^{2}$, reduces the influence of the flow velocity on the fibres' orientation.

- Meanwhile, the fibres distribution is more isotropic towards the centre of the vortex.

Finally, the present work also investigates the impact of $R e$ on the vortex size for $\phi a_{r}^{2}=12$. Numerical observation by Fig. 12 shows that the length of vortex lightly reduces with the increasing of $R e$ for a range of $R e \in\{0,1,2,5\}$. This is in good agreement with those in Abdul-Karem et al. [26].

\subsection{The 1:4 axisymmetric expansion flow}

The geometry of 1:4 axisymmetric expansion flow is schematically described in Fig. 13 in which $L_{D}=4$ and $R_{D}=1$ are the length and radius of the downstream tube, respectively; $L_{U}=6$ and $R_{U}=0.25$ the length and radius of the upstream tube, respectively; and $L_{v}$ the vortex length. The expansion ratio $(\beta)$ and dimensionless vortex length $\left(L_{v}^{*}\right)$ are defined as in Eq. (27) but for the expansion flow. The similar problem was studied by [11] using the finite-element method-based BCF. The boundary conditions of this problem are described as follows.

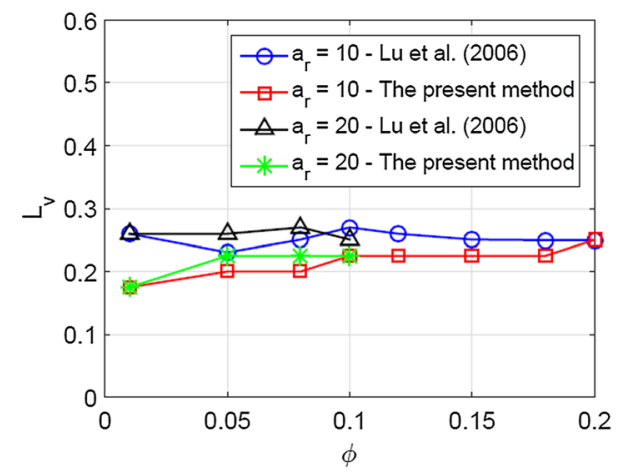

Fig. 15 1:4 axisymmetric expansion flows of fibre suspensions: the influence of fibre parameters on the vortex length $\left(L_{\mathrm{v}}{ }^{*}\right)$ for $a_{r}=10$ coupled with a range of $\phi €\{0.01,0.02,0.05,0.08,0.10,0.12,0.15,0.18,0.20\}$; and for $a_{r}=20$ coupled with a range of $\phi \in\{0.01,0.02,0.05,0.08,0.10\}$

(i) At the inlet $\overline{O A}$, the stream function, vorticity and velocity, are set up using the values at the outlet of the axisymmetric contraction flow investigated in Sect. 4.2. $\frac{\partial \Psi}{\partial \mathrm{z}}=0$;

(ii) At the outlet $\overline{D E}$, the stream function, vorticity and velocity at the inlet of the axisymmetric contraction problems are used as the Dirichlet boundary conditions of this problem. $\frac{\partial \Psi}{\partial z}=0$;

(iii) On the walls $\overline{A B}, \overline{C D}$ and $\overline{B C}$, non-slip boundary condition is set up for the velocity: $u_{z}=0$ and $u_{r}=0$. Hence, the corresponding boundary conditions for the stream function and the vorticity on the walls are set up as follows.

- $\Psi=0, \frac{\partial \Psi}{\partial x}=0$ and $\omega=\omega_{w_{1}}$ on the wall $\overline{A B}$;

- $\Psi=0, \frac{\partial \Psi}{\partial z}=0$ and $\omega=\omega_{w_{2}}$ on the wall $\overline{B C}$;

- $\Psi=0, \frac{\partial \Psi}{\partial r}=0$ and $\omega=\omega_{w_{3}}$ on the wall $\overline{C D}$; where $\omega_{w_{1}}$, $\omega_{w_{2}}$ and $\omega_{w_{3}}$ are determined using Eq. (11) with the known stream function at each time step;

(iv) On the centreline $\overline{O E}$, the symmetric boundary condition for the velocity is applied, i.e., $\frac{\partial u_{z}}{\partial r}=0$ and $u_{r}=0$. The corresponding boundary conditions for the stream function and vorticity are set up as $\Psi=\Psi_{c}, \frac{\partial \Psi}{\partial \mathrm{r}}=0$ and $\omega=0$, where $\Psi_{c}$ is determined by Eq. (10) using the inlet boundary condition of the velocity.

A non-uniform grid is designed for the simulation. Finer grids in regions near the outlet and inlet and around the abrupt expansion and the centreline are necessary to produce accurate solutions. A detailed grid is described in Fig. 14 and detailed as follows:

- On axial direction: $\Delta z_{1}=0.05 \forall z \in[5,7], \Delta z_{2}=0.1$ $\forall z \in[0,5] \bigcup[7,10]$; 


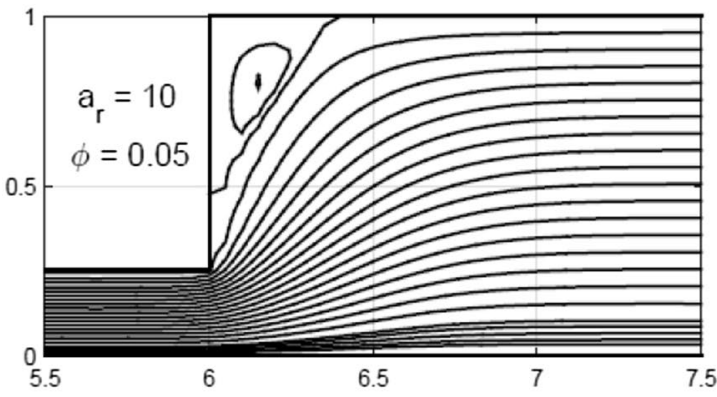

(a)

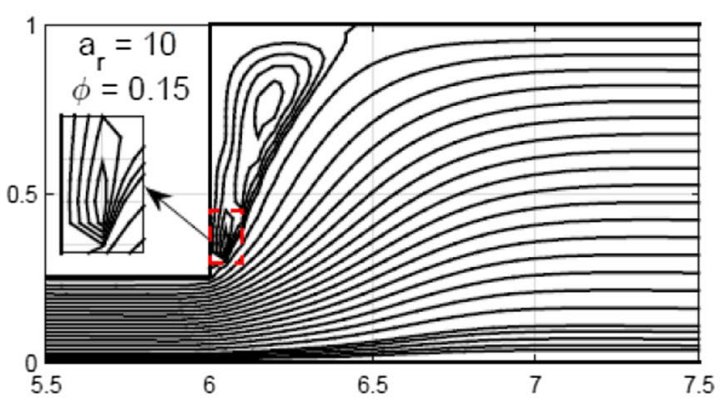

(c)

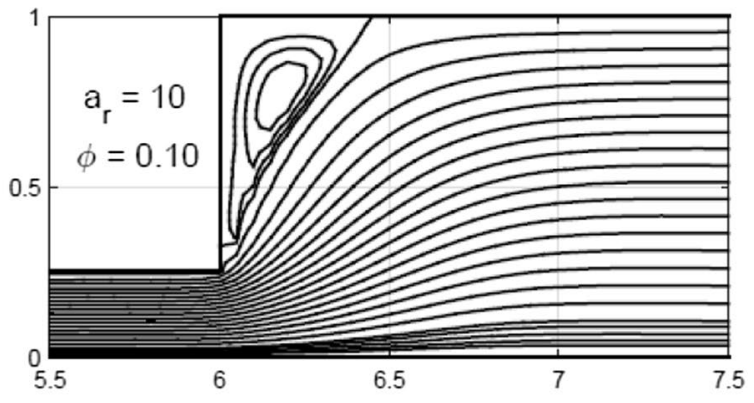

(b)

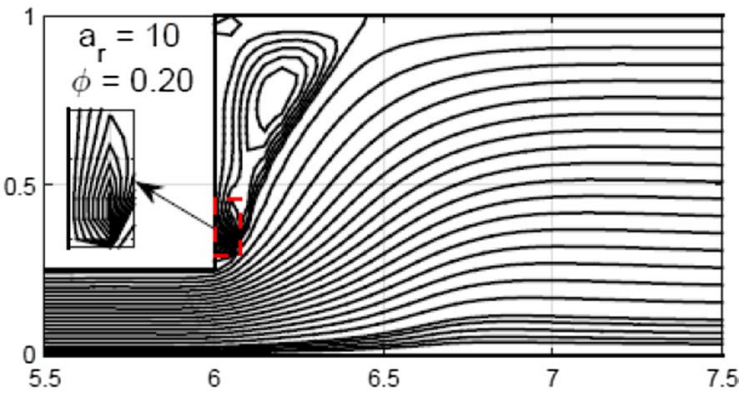

(d)

Fig. 16 1:4 axisymmetric expansion flows of non-dilute suspensions: the influence of $\phi a_{r}^{2}$ on the salient vortex pattern for $\phi a_{r}^{2}=\{5,10,15,20\}$. A lip vortex was observed for higher values of $\phi a_{r}^{2}$

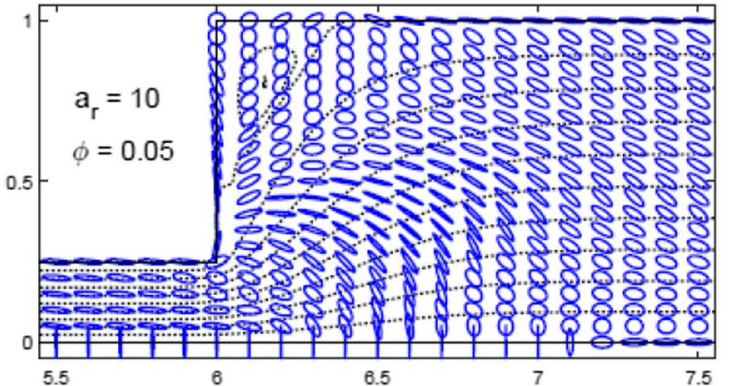

(a)

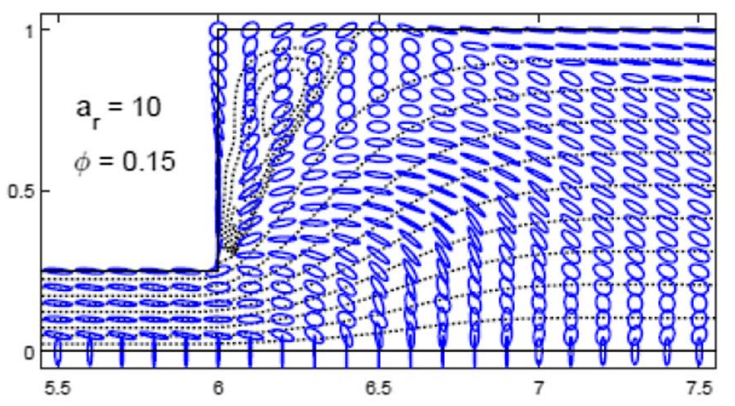

(c)

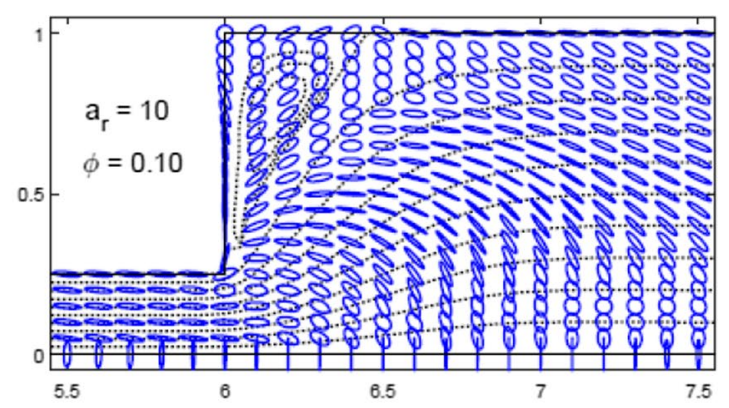

(b)

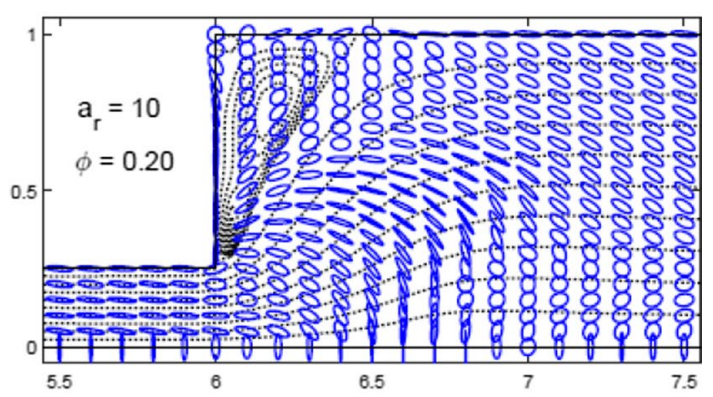

(d)

Fig. 17 1:4 axisymmetric expansion flows of non-dilute suspensions: the fibre distribution around the expansion area for $a_{r}=10$ and $\phi \in\{0.05,0.10,0.15,0.20\}$ 
Fig. 18 1:4 axisymmetric expansion flows of non-dilute suspensions: the effect of the Reynold number on the salient corner vortex for $a_{r}=10$ and $\phi=0.12$

Fig. 19 1:4 axisymmetric expansion flows of non-dilute fibre suspensions for $a_{r}=10$ and $\phi=0.15$ : the evolution of fibre stresses including shear stress $\left(\tau_{f}^{z r}\right)$, normal stresses $\left(\tau_{f}^{z z}\right.$ and $\left.\tau_{f}^{r r}\right)$ and the first normal stress difference $\left(\tau_{f}^{r r}-\tau_{f}^{z z}\right)$ at the position $z=5.75$ and $r=0.5$

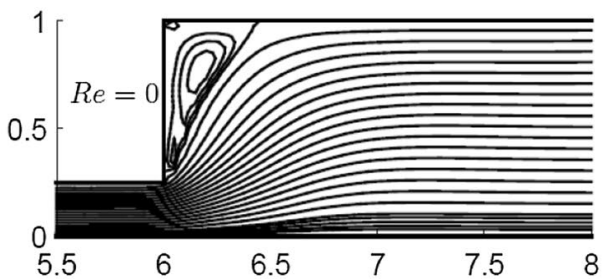

(a)

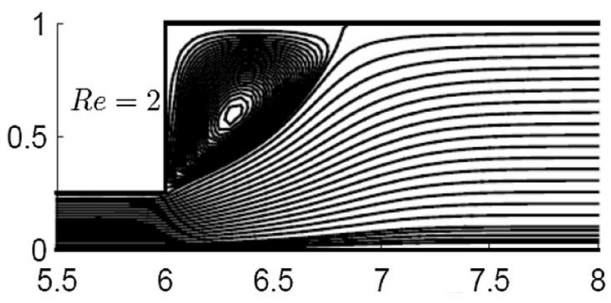

(c)

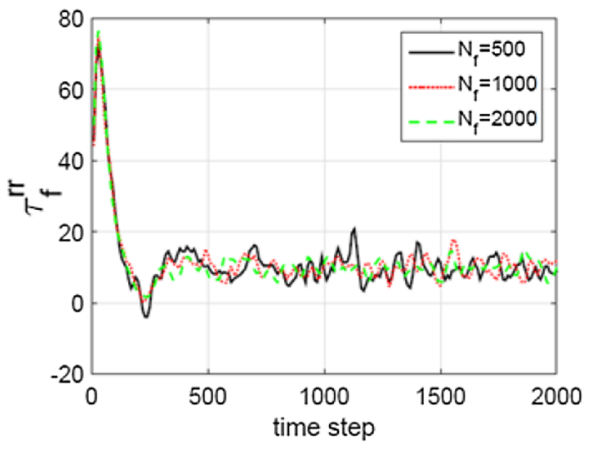

(a)

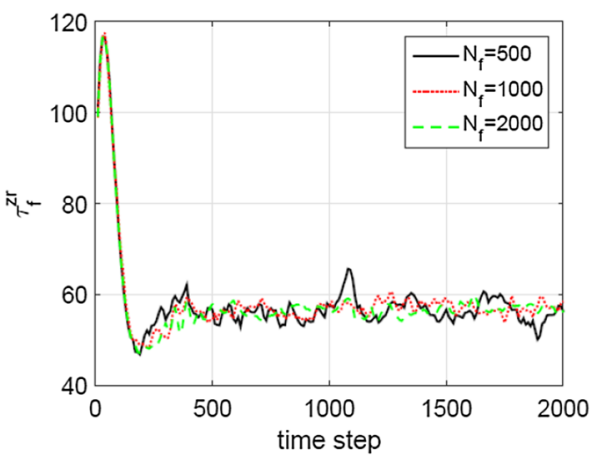

(c)

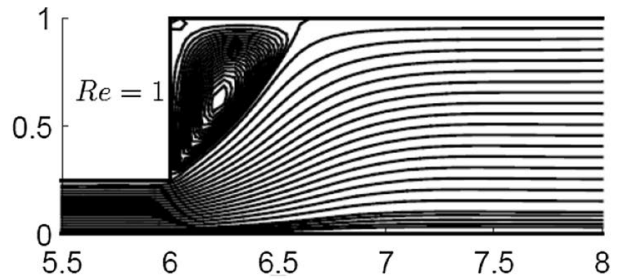

(b)

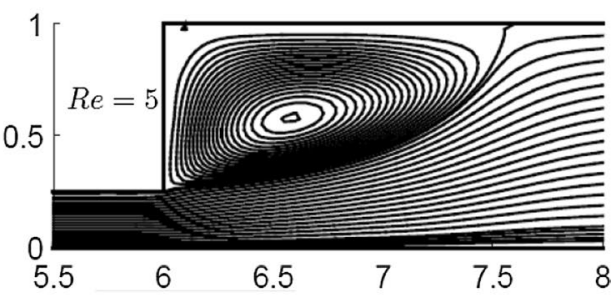

(d)

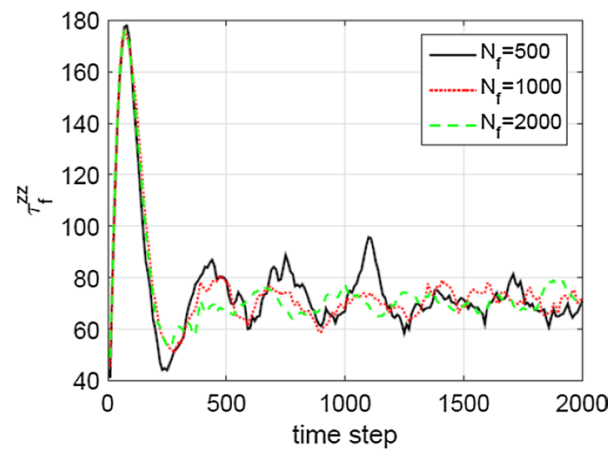

(b)

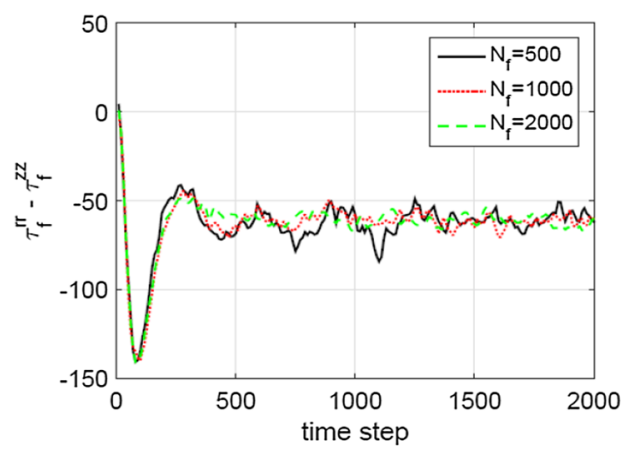

(d)
- On radial direct: $\Delta r_{1}=0.01 \forall r \in[0,0.1]$; and $\Delta r_{2}=0.025 \forall r \in[0.1,1]$.

The time step size $\Delta t=1 E-3$ is also used in this simulation as in the contraction flow problem. However, a smaller time step is used at the initial time for the simulation of several highly concentrated suspensions. Numerical results by the present method are discussed and compared with those by [11] and other publications as follows:

The effect of fibre parameters $\left(\phi, a_{r}\right)$ on the vortex length $\left(L_{v}^{*}\right)$ of the 1:4 expansion flow is presented by Fig. 15 for two cases of fibre suspensions: (i) $a_{r}=10$ coupled with a range of $\phi \in\{0.01,0.02,0.05,0.08,0.10,0.12,0.15,0.18,0.20\} \quad$; 
and (ii) $a_{r}=20$ coupled with a range of $\phi \in\{0.01,0.02,0.05,0.08,0.10\}$. The numerical results by Fig. 15 are in very good agreement with those by [11]. Indeed, in the contraction flows the vortex length is more pronounced with increasing $\phi$ and/or $a_{r}$ (Fig. 10), while it changes insignificantly in the expansion flows $\left(L_{v}^{*} \approx 0.18 \ldots 0.26\right)$.

Moreover, numerical results by the present method confirm that the vortex lengths in the expansion flows are smaller than those in the contraction flows with the same fibre parameters. However, while the vortex length is nearly unchanged, a small lip vortex is observed near the re-entrant corner since a sufficiently large value of $\phi a_{r}^{2}(15 \& 20)$ as shown in Fig. 16 for a range of $\phi a_{r}^{2}=\{5,10,15,20\}$. This observation is notable, because lip vortex was reported only for the Newtonian and viscoelastic expansion flows with high expansion ratio and Weissenberg number by Baloch and Webster [5] and Baloch et al. [27] but not in any report on fibre suspension flows.

The orientation of fibres as well as streamlines (black dashed lines) around the expansion area for a range of $\phi a_{r}^{2}=\{5,10,15,20\}$ is shown in Fig. 17. Results show that except fibres on the centreline, most fibres align with the flow direction (straight line or ellipse) in the upstream region, whereas the direction of fibres is more or less random along the streamline (a circle/circular ellipse) in the downstream region. In other words, near the vortex boundary fibres are not tangential to streamlines, which is in contrast to our numerical observation in the simulation of contraction flow (see Fig. 11). These findings are in good agreement with those of several numerical and experimental works Abdul-Karem et al. [26], Chiba and Nakamura [28] and Verweyst and Tucker [29].

The significant impact of inertia on the vortex size is also found by the present method. Indeed, results by Fig. 18 show a fast development of vortex with increasing $R e=\{0,1,2,5\}$, which was reported in several experimental works by Abdul-Karem et al. [26] and Townsend and Walters [30]. Furthermore, the observation also shows, while the vortex boundary is convex referred to the centreline for the creeping flow ( $R e=0$, Fig. 18a), it is gradually concave for the flows at higher $R e$ (Fig. 18b-d).

A simulation of the 1:4 expansion flow of non-dilute fibre suspensions with the fibre's aspect ratio $a_{r}=10$ and volume fraction $\phi=0.15$ using a range of the numbers of fibre configurations $N_{f}=\{500,1000,2000\}$ is also carried out to study the impact of the fibre configuration on the mechanical properties of suspension. Figure 19 presents the profiles of shear stress $\left(\tau_{f}^{z r}\right)$, normal stresses $\left(\tau_{f}^{z z}\right.$ and $\left.\tau_{f}^{r r}\right)$ and the first normal stress difference $\left(\tau_{f}^{r r}-\tau_{f}^{z z}\right)$ at the position $(\mathrm{z}, \mathrm{r})=(5.75,0.5)$ in the considered domain. The numerical results indicate that while $\tau_{f}^{r r}$ and $\tau_{f}^{z r}$, about 12 (Fig. 18a) and
59 (Fig. 18c), respectively, are in good agreement with those of the similar problem by Verweyst and Tucker [11], $\tau_{f}^{z z}$ and $\tau_{f}^{r r}-\tau_{f}^{z z}$ reported in Fig. 18b, d are smaller than those mentioned in the same publication. In addition, the smoothness of curve presenting fibre stresses, especially $\tau_{f}^{z z}$, is improved with increasing the number of fibre configurations as described in Fig. 19.

\section{Conclusions}

The multiscale simulation method based on the combination of high-order integral RBF approximation and BCF idea is further developed for non-dilute fibre suspensions in this work. The research is to simulate semi-dilute and concentrated fibre suspension flows in which the evolution of fibre configurations governed by the Folgar-Tucker equation are determined by the BCF method and the fibre stress is approximated by the Phan-Thien-Graham model. The efficiency of the present method for the simulation of nondilute fibre suspension flows is based on both the accuracy of the method and the stability of the stochastic process. As an illustration of the present method, two challenging flows: the 1:4 axisymmetric expansion and 4:1 axisymmetric contraction flows are investigated for a large range of the fibre parameters from semi-dilute to concentrated regimes. Numerical results by the present method are in good agreement with those published by Lu et al. [11]. Moreover, several dynamics behaviours of suspension flows by the present work including the orientation of fibres are nearly similar to experimental observations published in Abdul-Karem et al. [26], Verweyst and Tucker [29] and Baloch et al. [5]. Finally, the present work finds the existence of a lip vortex in the 1:4 expansion flows of highly concentrated fibre suspensions.

Funding Open Access funding enabled and organized by CAUL and its Member Institutions.

Open Access This article is licensed under a Creative Commons Attribution 4.0 International License, which permits use, sharing, adaptation, distribution and reproduction in any medium or format, as long as you give appropriate credit to the original author(s) and the source, provide a link to the Creative Commons licence, and indicate if changes were made. The images or other third party material in this article are included in the article's Creative Commons licence, unless indicated otherwise in a credit line to the material. If material is not included in the article's Creative Commons licence and your intended use is not permitted by statutory regulation or exceeds the permitted use, you will need to obtain permission directly from the copyright holder. To view a copy of this licence, visit http://creativecommons.org/licenses/by/4.0/. 


\section{References}

1. Jeffery GB (1922) The motion of ellipsoidal particles immersed in a viscous fluid. Proc R Soc 102(715):161-179

2. Folgar F, Tucker CL (1984) Orientation behaviour of fibers in concentrated suspensions. J Reinf Plast Compos 3(2):98-119

3. Lipscomb GG, Denn MM, Hur DU, Boger DV (1988) The flow of fiber suspensions in complex geometries. J Non-Newt Fluid Mech 26(3):297-325

4. Chiba K, Nakamura K, Boger DV (1990) A numerical solution for the flow of dilute fiber suspensions through an axisymmetric contraction. J Non-Newt Fluid Mech 35(1):1-14

5. Baloch A, Webster MF (1995) A computer simulation of complex flows of fibre suspensions. Comput Fluids 24(2):135-151

6. Advani SG, Tucker CL (1987) The use of tensors to describe and predict fiber orientation in short fiber composites. J Rheol 31(8):751-784

7. Hulsen MA, Van Heel APG, Van Den Brule BHAA (1997) Simulation of viscoelastic flows using Brownian configuration fields. J Non-Newt Fluid Mech 70(1):79-101

8. Tran-Canh D, Tran-Cong T (2002) Computation of viscoelastic flow using neural networks and stochastic simulation. KoreaAustralia Rheol J 14(4):161-174

9. Tran-Canh D, Tran-Cong T (2004) Element-free simulation of dilute polymeric flows using Brownian configuration fields. Korea-Australia Rheol J 16(1):1-15

10. Fan XJ, Phan-Thien N, Zheng R (1999) Simulation of fibre suspension flows by the Brownian configuration field method. J Non-Newt Fluid Mech 84(2-3):257-274

11. Lu Z, Khoo BC, Dou H-S, Phan-Thien N, Seng Yeo K (2006) Numerical simulation of fibre suspension flow through an axisymmetric contraction and expansion passages by $\mathrm{BCF}$ method. Chem Eng Sci 61(15):4998-5009

12. Dou H-S, Khoo BC, Phan-Thien N, Yeo KS, Zheng R (2007) Simulations of fibre orientation in dilute suspensions with front moving in the filling process of a rectangular channel using level-set method. Rheol Acta 46(4):427-447

13. Eberle APR, Vélez-García GM, Baird DG, Wapperom P (2010) Fiber orientation kinetics of a concentrated short glass fiber suspension in startup of simple shear flow. J Non-Newt Fluid Mech 165(3):110-119

14. Tran CD, Phillips DG, Tran-Cong T (2009) Computation of dilute polymer solution flows using BCF-RBFN based method and domain decomposition technique. Korea-Australia Rheology J 21(1):1-12

15. Tran CD, An-Vo D-A, Mai-Duy N, Tran-Cong T (2011) An integrated RBFN-based macro-micro multi-scale method for computation of visco-elastic fluid flows. C Comput Model Eng Sci 82(2):137-162
16. Nguyen HQ, Tran CD, Tran-Cong T (2015) RBFN stochastic coarse-grained simulation method: part I-dilute polymer solutions using bead-spring chain models. C Comput Model Eng Sci 105(5):339-439

17. Nguyen HQ, Tran CD, Tran-Cong T (2015) A multiscale method based on the fibre configuration field, IRBF and DAVSS for the simulation of fibre suspension flows. C Comput Model Eng Sci 109(4):361-403

18. Tran CD, Mai-Duy N, Le-Cao K, Tran-Cong T (2012) A continuum-microscopic method based on IRBFs and control volume scheme for viscoelastic fluid flows. C Comput Model Eng Sci 85(6):499-519

19. Phan-Thien N, Graham AL (1991) A new constitutive model for fibre suspensions: flow past a sphere. Rheol Acta 30(1):44-57

20. Kitano T, Kataoka T, Shirota T (1981) An empirical equation of the relative viscosity of polymer melts filled with various inorganic fillers. Rheol Acta 20(2):207-209

21. Bird RB, Armstrong RC, Hassager O (1987) Dynamics of polymeric liquids, vol 1. Wiley, New-York

22. Bird RB, Curtiss CF, Armstrong RC, Hassager O (1987) Dynamics of polymeric liquids, vol 2. Wiley, New-York

23. Phan-Thien N, Fan X-J (1999) Pressure drop created by a sphere settling in a tube containing a fiber suspension. J Rheol 43:1

24. Mai-Duy N, Le-Cao K, Tran-Cong T (2008) A Cartesian grid technique based on one-dimensional integrated radial basis function networks for natural convection in concentric annuli. Int J Numer Meth Fluids 57(12):1709-1730

25. Thai-Quang N, Mai-Duy N, Tran C-D, Tran-Cong T (2012) High-order alternating direction implicit method based on compact integrated-RBF approximations for unsteady/steady convection-diffusion equations. C - Comput Model Eng Sci 89(3): 189-220

26. Abdul-Karem T, Binding DM, Sindelar M (1993) Contraction and expansion flows of non-newtonian fluids. Compos Manuf 4(2):109-116

27. Baloch A, Townsend P, Webster MF (1996) On vortex development in viscoelastic expansion and contraction flows. J NonNewt Fluid Mech 65(2):133-149

28. Chiba K, Nakamura K (1998) Numerical solution of fiber suspension flow through a complex channel. J Non-Newt Fluid Mech 78(2):167-185

29. Verweyst BE, Tucker CL (2002) Fiber suspensions in complex geometries. Can J Chem Eng 80(6):1093-1106

30. Townsend P, Walters K (1994) Expansion flows on non-Newtonian liquids. Chem Eng Sci 49(5):748-763

Publisher's Note Springer Nature remains neutral with regard to jurisdictional claims in published maps and institutional affiliations. 